\title{
ARTIFICIAL NEURAL NETWORKS FOR MEDICAL DIAGNOSIS: A REVIEW OF RECENT TRENDS
}

\author{
Egba. Anwaitu Fraser ${ }^{1}$, Okonkwo, Obikwelu R ${ }^{2}$ \\ ${ }^{1}$ Department of Computer Science, School of Science Education (SSE), Federal College \\ of Education (Technical), PMB 11, Omoku, Rivers State, Nigeria \\ ${ }^{2}$ Department of Computer Science, Faculty of Natural Sciences, NnamdiAzikiwe \\ University, Awka, Anambra State, Nigeria
}

\begin{abstract}
Artificial Intelligence systems (especially computer-aided diagnosis and artificial neural networks) are increasingly finding many uses in medical diagnosis application in recent times. These methods are adaptive learning algorithms that are capable of handling multiple and heterogeneous types of clinical data with a view of integrating them into categorized outputs. In this study, we briefly review and discuss the concept, capabilities, and applicability of artificial neural network techniques to medical diagnosis, through consideration of some selected physical and mental diseases. The study focuses on scholarly researches within the years, 2010 to 2019. Findings show that no electronic online clinical database exists in Nigeria and the Sub-Saharan countries, most review researches in this area focused mainly on physical diseases without considering mental illnesses, the application of ANN in mental and comorbid disorders have not been thoroughly studied, ANN models and algorithms consider mainly homogeneous input data sources and not heterogeneous input data sources, and ANN models on multi-objective output systems are few as compared to single output ANN models.
\end{abstract}

\section{KEYWORDS}

Medical diagnosis, Artificial intelligence, Artificial neural networks, Feed-forward backpropagation, Convolutional Neural Network, diabetes, cardiovascular, cancer, malaria, and Mental Disorder

\section{INTRODUCTION}

Diagnosis is one of the major tasks of all physicians and its importance to man cannot be overemphasized. In diagnosing, physicians are challenged with the task of deducing certain diseases or formulating a treatment based on specified signs and symptoms or observations and knowledge. Zagorechi et al. [1] define diagnosis as "the process of assigning a label to an illness or other problems by examining observations and symptoms". Simple as stated, diagnosis is classifiedby scholars as a rigorous, complex and multifaceted task that is bedeviled with multiple challenges. Some of such challenges include having symptoms of a disease(s) that are not specific to only one disease and sometimes overlapping with the symptoms of other diseases [2]; having an unclear or inadequate description of how and what a patient feels in the body as a result of a loss of memory or voice; having severe mental conditions that distort cognitive abilities; frequency of the occurrence of diseases; and risk factors such as age, sex, and body mass index (BMI) that influences both the structure of dependencies between symptoms and illnesses. Others include wrong and untimely interpretation of information provided by patients, and unnecessary delays and errors in the analysis of laboratory results [3], lack of facilities, poor technical know-how and the imbalanced ratio of patients to doctors in healthcare especially in the developing countries, etc. No wonder, [4] asserts that $50 \%$ of diagnoses are wrong. 
Over the years, several positive advancements have been recorded in the field of a medical diagnosis from antiquity to modern times. In all, however, traditionally, the wealth of experience of physicians over years in service plays a significant role in ineffective medical diagnosis. This experience depends largely on the intuitive statistical analysis of situations by the physician to arrive at diagnostic solutions. For example, reports show that the illness AIDS which showed by a bunch of infections and cancer (malignant growth) states was not found straightforwardly by doctors however by statistical specialists who were watching the implausible density of uncommon disease cases at the US west coast [5]. This is in support of the attestation by Buch et al. [6] that"informing clinical decision making through insights from past data is the essence of evidence-based medicine". Nonetheless, there are numerous deficiencies of measurable estimation methods such as statistics, one of which is that quality cannot be guaranteed when managing deficient or incomplete, noisy and non-linear data items like clinical data [7]. Notwithstanding, besides the statistical measurable procedures, two universally accepted disease classification techniques, which include specifically: International Classification of Diseases tenth Revision edition (ICD - 10), and the Diagnostic and Statistical Manual of Mental Disorders-IV edition (DSM - IV) are frequently used [8]. They are standard universally accepted disease classifiers.

In recent times, artificial intelligence (AI) has increased the chances of physicians with little or no statistically experienced to apply the benefits of artificial intelligence-based diagnostic approaches to enhance service improvements [6], by providing techniques that uncover complex associations which cannot be reduced to an equation. Artificial Intelligence (AI) approaches, provide reasoning capability, which consists of inferences from facts and rules using heuristics, pattern matching or other search approaches [9] and has contributed significantly to the evolution of biomedicine and medical informatics [10]. Recent areas of development in AI in relationship to medical diagnostics which are the leading methods with which physicians are assisted in this demanding task include - the expert system [11], [12], [13], fuzzy logic [14], Artificial Neural Networks [15] and neuro-fuzzy expert system [16], [17].

This study aims at reviewing selected scholarly researches that are related to the application of Artificial Neural Network (ANN) techniques to the diagnosis of diseases in patients. Specifically, the focus is on relevant works of literature that fall within the years 2010 to 2019. After a description of the basic elements of ANN and its operations, its application in medicine and potential future trends are examined.

\section{MATERIAL AND MethodS}

A search criterion was designed for the extraction of relevant literature on research works regarding ANN in medical diagnosis from three (3) selected online scientific electronic opensource libraries namely "Science Direct", "Microsoft Academic", and "IEEE Transaction". The search was based on the query strings "Artificial Neural Networks", "Artificial Neural Network in Medical Diagnostics" and "Artificial Neural Networks in Mental Disorder Diagnosis" which produced approximately 2000 articles. The abstracts of the articles were first reviewed to eliminate researches that are not relevant to the topic. Thereafter, the full text of the articles was meticulously reviewed to further eliminate articles that are not relevant to the study. The criteria used was as follows:

i. Only articles published in ANN or medical diagnostic related journals were selected;

ii. Only articles that clearly describe how the mentioned ANN techniques could be applied, and (or) improve medical diagnosis were selected; 
iii. Conference papers, textbooks, masters and doctoral dissertations, and unpublished working papers were excluded. This is due to the report by [18] that journals are the leading source and reference of researchers.

After a thorough analysis of the selected articles, the collected notes were organized into two sections as the Concept of Artificial Neural Networks, and a review of artificial neural networks in medical diagnostics.

\section{Conceptual Framework of Artificial Neural Networks}

Artificial Neural Network (ANN) is a computational model and an information processing paradigm that is inspired by the way biological nervous systems, such as the brain, process information [19]. Amato et al. [20] opine that ANN "is a mathematical representation of the human neural architecture, reflecting its "learning", "generalization" and "abstraction" abilities". An artificial neural network consists of various layers of interconnected "neurons" (or "nodes") called processing elements (PEs). ANNs consist of a small number of neurons or processing elements (PEs) says from tens to thousands of PEs. Each neuron or PE in a layer is connected with each neuron or PE in the next layer through a weighted connection. The value of the weight $\left(\mathrm{w}_{\mathrm{ij}}\right)$ is an indication of the strength of the linking between the neuron (i-th) or PE in a layer and neuron (j-th) or PE in the next layer [20]. In each layer, there is a finite number of nodes with activation functions. The model receives data items by the processing elements (PEs) in the input layer and transfers them to the processing elements (PEs) in the first hidden layer through the weighted links. The data items received at this point are mathematically processed and the results are transferred to the next layer and so on until they get to the output layer which is the last layer. And finally, the output is assembled at the output layer as shown in fig. 1.

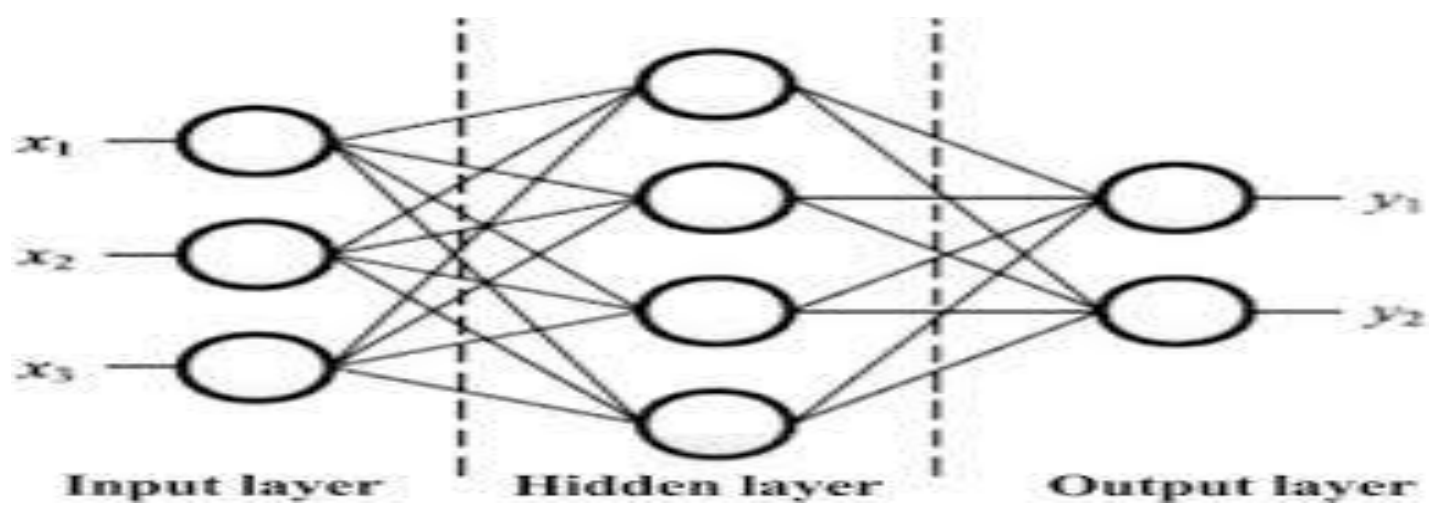

Fig.1. Artificial Neural network structure and layers (Sourced from: [20])

There are different architectural structures of ANN of which the most common is the multilayer perceptron (MLP). The MLP and many other variations of ANN, learn by using an algorithm known as the backpropagation. The backpropagation allows input data to be repeatedly presented to the neural network. The structure is designed such that the first layer is the input layer and the last layer is the output layer. Between the input and the output is an additional one or more layers of units called hidden layers. The number of neurons or PEs in a layer and the number of layers are strongly dependent on the complexity of the system studied. Thus, the determination of the optimal network architecture is necessary. 


\section{Application of artificial Neural networks in Medical DIAGNOSIS}

The concept of the "application of artificial neural networks in medical diagnostics" was pioneered by [21] in their article titled, "Artificial intelligence in medical diagnosis" and has since attracted much attention. Several research papers spread across diverse fields of medical sciences have been published among which [22], [21], [23] and so on. In almost all the reviews, the emphasis was placed on physical diseases, leaving out mental and social illnesses. Suffice it to say that [24] defines health as "a state of complete physical, mental and social well-being and not merely the absence of diseases and infirmities". Thus, a healthy human must be both physically and mentally and socially sound in health. In this research, we review the application of artificial neural network techniques to medical diagnosis on some selected physical and mental disorders.

\subsection{Diabetes}

Diabetes is a serious health problem that is common in developed countries [25]. The disease is on the increase with an estimated number reaching 425 million cases globally, 16 million cases in the African region, and about 1,731,811 cases in Nigeria as of 2015, according to the International Diabetes Federation [26]. The World Health Organization [24] predicts and projects that it may become the 7th leading cause of death in 2030 with the rate of its increase. Diabetes occurrence is a result of an increased level of glucose in the human body system due to the absence or inadequate amount of insulin in the human body. Artificial Neural Networks have been successfully trained to solve the problem of its diagnosis. Notable is the research of Alade et al. [27], where they developed and trained an ANN model for the diagnosis of diabetes mellitus in pregnant women. They developed a four-layer Artificial Neural Network, trained the model using two algorithms, that is, the back-propagation and the Bayesian Regulation (BR) algorithms. The model consists of eight (8) neurons in the input layer, two hidden layers with ten (10) neurons each, and a neuron in the output layer which represents the diagnosis result. The dataset was gathered from a medical database called the Prima Indian Database set. It contained 768 rows of data and the tests on the individuals used were on their blood pressure, Triceps Skin Thickness, Insulin, Body Mass Index, Diabetes Pedigree Function, and age. 500 people tested positive and 268 people tested negative giving an accuracy level of $92 \%$ after validation by regression. [28] also developed a diagnostic model using three (3) variations of ANN (Bayesian Regulation Algorithm(BRA), J48 \& Radial Basis Function - Neural Networks(RBF-NN)) from a sample of data collected from 768 respondents collected from the Pima Indian Database. The diagnostic accuracy rate for BRA proved to be $79,95 \%$, that of $\mathrm{J} 48$ yielded $76.52 \%$ and RBF-NN yielded $74.34 \%$.

\subsection{Cardiovascular Disease}

Cardiovascular diseases (CVDs) according to Szolovits et al. [21] involve diseases " that affect the heart or blood vessels, both arteries, and veins". CVDs include coronary heart disease (disease of the blood vessels supplying the heart muscle); cerebrovascular disease (that affects blood vessels supply to the brain); peripheral arterial disease (affects the supply of blood vessels to the arms and legs); rheumatic heart disease (causes damage to the heart muscles and heart valves from rheumatic fever triggered by streptococcal bacteria); congenital heart disease(causes abnormalities in the formation of heart structure present at birth); and deep vein thrombosis and pulmonary embolism (caused by blood clots in the veins of the legs, which can extricate and move to the heart and lungs). 
It is one of the leading causes of death in several countries with an estimate of 17.9 million people dying annually representing $31 \%$ of global deaths. Literature abounds as to the success of the application of ANN to the training of data for the analysis and diagnosis of this disease with a high degree of success. [15] used Feed forward Back Propagation Neural Networks (FFBP-NN) to diagnose Acute Nephritis \& Heart disease with and an accuracy rate of $99 \%$ and $95 \%$ respectively. Myers et al [29] used ANN with Firefly(FF), multilayer Perceptron neural networks(MPNN) and Back Propagation(BP) to diagnose CVD and it yielded $72 \%$ on testing the model on data collected from Va Palo Alto Healthcare. [30], and [31] developed ANN and DNN respectively to diagnose Coronary heart Disease. The former collected 487 samples of data from the Central Clinical Hospital of Russian Railway and the later collected data samples of 303 from the Cleveland Clinic Foundation, New York, USA. Accuracy results were (64 - 94)\% and 83.67\% respectively. [32] and [33] also used CNN to diagnose Myocardial Infarction and Deep Convolutional Neural Networks (DCNN) to diagnose Heartbeat rhymes. The 2017a study yielded an accuracy rate of $93.53 \%$ for original and $95.22 \%$ for noise-free electroencephalograms(ECGs) respectively while the [32] study yielded an accuracy rate $94.03 \%$ and $93.47 \%$ for original and noise-free ECGs respectively.

\subsection{Cancer Disease}

Reports regarding the estimation of cancer diseases by the International Agency for Research on Cancer (IARC) published in the American Cancer Society (www.cancer.org) shows that in 2018 alone, there were about 17 million new cancer cases and 9.5 million cancer deaths over the globe. IARC also projects that by 2040, the global burden of cancer cases will be expected to grow to 27.5 million new cancer cases and 16.3 million cancer deaths due to the growth and aging rate of the population. The future burden will probably be even larger due to the increasing prevalence of factors that increase risks, such as smoking, unhealthy diet, physical inactivity, and fewer childbirths, in economically transitioning countries. ANN models have successfully been applied in the solving of problems of the diagnosis of cancer worldwide. Notable among them is the works of [34], [35], [36], [37] , [38], etc. In the research of Pergialiotis et al. [38], they investigated the diagnostic accuracy of three different techniques, that is, logistic regression, ANNs, and the Classical and Regression Tree(CART) for the prediction of endometrial cancer in postmenopausal women with vaginal bleeding or endometrial thickness $5 \mathrm{~mm}$, as determined by ultrasound examination. It is a case-control study based on data obtained from the analysis of pathology reports of curettage specimens in postmenopausal women. They employed the classical regression analysis, ANN and CART analysis using the IBM SPSS and MATLAB statistical packages. Overall, 178 women were enrolled. Among the women, 106 of them were women diagnosed with carcinoma, whereas the remaining 72 were women that had normal histology in the final specimen. In their performances, ANN analysis performed better with a sensitivity of $86.8 \%$, a specificity of $83.3 \%$, and an accuracy rate of $85.4 \%$. The CART analysis also performed well with a sensitivity of $78.3 \%$, a specificity of $76.4 \%$, and an accuracy level of $77.5 \%$. However, the performance of regression analysis poor as compared to the other two with a sensitivity of $76.4 \%$, a specificity of $66.7 \%$, and an accuracy rate of $72.5 \%$.

\subsection{Malaria}

In a world malaria report of 2017 that draws on data from 91 countries in the globe and areas with ongoing malaria transmission, the world health organization in [24] estimated 216 million cases of malaria occurred worldwide (95\% CI: 196 - 263 million), an increase of 5 million cases over $2015.90 \%$ of the malaria cases were in the WHO African regions followed by $7 \%$ by the Who Southeast Asia Region and the WHO Eastern Mediterranean Region with 2\%. It is also reported that $80 \%$ of the global malaria burden cases and 15 countries out of the 91 countries reporting indigenous malaria cases are from sub-Saharan Africa. The World malaria report of 
2017 estimates that 445,000 deaths from malaria will happen globally and the WHO African Region accounted for $91 \%$ of all malaria deaths in 2016. The ANN model has been applied for the diagnosis of malaria disease successfully. Ziynet \& Bozkurt [39] and Neha et al. [40] both worked on Anemia disease using Feed Back Neural Networks (FBNN), Deep Neural Networks (DNN), Probabilistic Neural Networks(PNN), Learning Vector Quantization(LVQ) and CNN with an accuracy result of $99.16 \%$ and $97.31 \%$ respectively. [23] and [41] worked on malaria diagnosis using MLP-BP and FFBP-NN with an accuracy yield of 85\% and 100\% respectively.

\subsection{Mental Disorders}

Mental Disorders (MD) are diseases characterized by persistently depressed mood, feelings of sadness or loss of interest in activities, causing significant impairment in daily life and have become one of the commonest causes of disabilities in the globe [24]. Artificial neural networks have recorded breakthroughs in the diagnosis of mental health problems such as depression, schizophrenia, bipolar, etc., [33] used the DCNN to diagnose seizure using EEG signal data collected from the Boon University Germany which yielded an accuracy rate and sensitivity rate of $88.67 \%$ and $95.00 \%$ respectively. In another study, [42] developed a diagnostic model using the BPNN for suicide attempt prediction with an accuracy rate for positive prediction value as $86.0 \%$ and negative predictive value as $84.1 \%$. Huang, $\mathrm{Wu}$, and $\mathrm{Su}$ [43] in their research proposed a domain adaptation technique that was based on a hierarchical spectral clustering algorithm. It was built to adapt a labeled emotion database into a collected unlabeled mood database for alleviating the data bias problem in an emotional space. A convolutional neural network $(\mathrm{CNN})$ with an attention mechanism was employed to produce an emotion profile (EP) of each speech response, elicited. The proposed model achieved an accuracy rate of $75.56 \%$, which gave an improved performance of $62.22 \%$ over support-vector-machine (SVM) and the CNN method gave 66.67\%. [44] developed a model for the diagnosis of Bipolar and Schizophrenia using the BPNN approach. This yielded an accuracy rate of $90 \%$ when tested with sociodemographic and biochemical data. [45] researched the diagnosis of Alzheimer's disease using the CNN model and [46] worked on dementia diagnosis, comparing the performance of 4 machine learning techniques namely J48, NB, RF \& MLP-NN. The accuracy rate, obtained after testing the performance on a dataset collected from the OASIS-brain Organization was J48 99.52\%, NB - 99.28\%, RF - 92.55\% and MLP-NN - 96.88\%. [47] studied the diagnosis of schizophrenia using the DDAN model and achieved an accuracy rate of $85.0 \%$ for multi-site pooling classification and $81.0 \%$ for leaving-site-out transfer classification.

\subsection{Depression and Comorbid Disease}

In [48], they applied the BPNN and ANFIS models to differentiate depression into grades. Of the two model types, ANFIS yielded better performance than BPNN on testing the models on datasets collected from various sources in India. [33] also studied the diagnosis of depression using the ANN. Using EEG signal data from the rights \& left hemisphere, the accuracy rates of 93.5\% and $86.0 \%$ respectively. It was shown that EEG from the right hemisphere is more distinctive in depression detection than those from the left hemisphere. [49] applied BN, BPNN, $\mathrm{SOV}, \mathrm{KNN}$, and FL to classify depression. They sourced data items from the University of Benin Teaching Hospital (UBTH) for the training of the model. They obtained accuracy results as follows: BN gave an accuracy rate of 0.975, BPNN yielded an accuracy level of 0.971, SVM yielded an accuracy of 0.916 , KNN yielded an accuracy level of 0.959 and FL gave an accuracy rate of 0.951 . Ojeme et al. [50] in another study considered depression and comorbid diseases using the MBNC model. Dataset consisted of 1090 patients of which 454 were males and 606 were females, from the University of Benin Teaching Hospital (UBTH). The accuracy rate was not measured but it was shown that predictive models have the potential of offering good performances to co-occurring disease in a patient. 
International Journal of Computer Science and Engineering Survey (IJCSES), Vol.11, No.3, June 2020

\section{DISCUSSION AND FINDINGS}

\section{The research showed that:}

i. There is no electronic online medical/clinical database library in Nigeria and other subSaharan countries. Medical data is sourced manually from hospitals and research agencies in developing countries like Nigeria. The few scholars who delved into this study either sourced their data directly from known hospitals using conventional data gathering methods such as interviews, observations, questionnaires or organized workshops where applicable[49],[17],[9], and [3], while others sourced clinical data from foreign database sources;

ii. Emphasis was placed on physical diseases like cancer, cardiovascular diseases, diabetes mellitus, etc. in most of the literature reviews by scholars on Artificial Neural Networks in Medical diagnostics paying little or no attention to mental and social disorders. Reviews involving mental disorders were targeted on a particular disorder and known focused on Africa and the sub-Saharan nations;

iii. No scholar has worked on the Application of ANN in Mental and comorbidity disorders. The few works in this area such as the use of Bayesian Networks by Ojeme, Akazue\&Nwelih[50] employed methodologies and algorithms such as Bayesian Networks and not ANN. In their research, they identified a gap in the literature and stated that "there is a gap in the literature on how the same techniques of machine learning could be used to simultaneously identify depression and comorbid physical illnesses"[50].

iv. The input datasets of most of the researches using ANN are from homogeneous sources such as symptoms, signs, images, voices, physiological, text messages and activities on social media. Known has considered multiple input sources, that is, heterogeneous sources of datasets.

v. Most of the works considered the diagnosis and prognosis of a particular disease at a time. The cases of multi-objective output, that is, diagnosis of multiple diseases given and input source lacks authoritative study.

vi. Combining multiple machine learning algorithms such as genetic algorithm, fuzzy logic, and artificial neural networks yield a better-improved accuracy rate.

\section{ConClusion}

This paper shows a growing interest in Artificial Neural Networks (ANN) that its models and algorithms are becoming standard tools in Computer science and especially in decision support and expert systems. ANN no doubt represents a powerful tool to help physicians and other medical experts and stakeholders perform diagnosis, prognosis, and other enforcements. The advantages of ANN which include:

i. Adaptability and flexibility of the systems;

ii. Ability to process a large volume of the dataset;

iii. Reduced likelihood of overlooking relevant information; 
iv. Timely diagnosis of diseases

v. Ability to process datasets collected from multiple sources like voices, images, symptoms, text messages, etc.

It makes it an important computational model in the development of modern clinical decision support and expert systems.

The studies reviewed here suggest that ANNS has proven to be suitable for a satisfactory medical diagnosis of both physical and mental illnesses. Their use has improved diagnostic accuracy and reliability thus increased patients' satisfaction. However, Guntuku et al. [51] , "ethical and legal questions about data ownership and protection, as well as clinical and operational questions about integration into systems of care" are some other challenges that require prompt addressing. Patients privacy protection is a major issue all over the world and needs to be addressed. Also, despite the widespread use of ANNs and other intelligent computational algorithms, the tool must be considered only as a facilitator of the final decision of a physician who holds the ultimate responsibility for critical evaluation of the ANN output.

\section{LiMITATIONS AND FUTURE STUDIES}

This research is limited to a few selected articles treating cases where ANN models are employed to the solving of the problem of diagnosis of diseases. The research did not consider the categorization of types of ANN techniques and the different hybrid methods that have been successfully applied to the solving of clinical diagnosis problems. Also, we did not delve into considering the multiple sources of data sources. The research concentrated on the number of successes achieved in the utilization of the ANN technique for medical diagnosis. Future works need to consider improving on these limitations and applying the scoping method and concentrate more on mental and social health problems as these are medical areas that are more difficult to diagnose.

\section{REFERENCES}

[1] Zagorechi A., Orzechowski P. \& Holownia K, "A System for Automated General Medical DIagnosis using Bayesian Networks.," Studies in Health Technology and Informatics, pp. DOI: 10.3233/978-161499-289-9-461 (ResearchGate), 2013.

[2] Kajan, S.; Pernecky, D.; and Goga, J., "Application of neural networks in medical diagnostics," Journal of Applied Biomedicine, pp. Vol. 11, issue 2, pp 47 -58. ISSN 1214-02887, 2013.

[3] Fashoto, S.G., Adeyeye M., Owolabi O. \& Odim M, "Modelling of the Feed Forward Neural Network with its Application in Medical Diagnosis," International Journal of Advances in Engineering \& Technology, pp. vol. 8, issue 4, pp 507 - 520., 2015.

[4] R. Brause, "Medical Analysis and DIagnosis by Neural Networks.".

[5] Miller, D.D. \& Brown, E.W, "Artificial Intelligence in Medical Practice: The question to the answer? " The American Journal of Medicine, pp. vol. 131, pp 129 - 133. https://doi.org/10.1016/j.amjmed.2017.10.035, 2018.

[6] Buch, V.H.; Ahmed, I.; and Maruthappu, M., "Artificial Intelligence in medicine: Current trends and future possibilities," British Journal of General Practice, pp. Vol. 68, Issue 668, pp 143 - 144. DOI: 10.3399/bjpx695213, 2018.

[7] Antonio, D., Jorge H. \& Paulo D.C, "Neural, Fuzzy and Neuro-fuzzy System for Medical Applications. In Intelligent and Adaptive Systems in Medicine," Series in Medical Physics and Biomedicine Engineering, Olivier C.L.H. \& Keith J.B.(Eds.). U.S.A: Taylor Francis Group, CRC Press, pp. pp127 - 170 .

[8] S. Bashyal, "Classification of Psychiatric Disorders using Artificial Neural Networks.," Advances in Neural Networks - ISNN, pp. pp 796 - 800., 2005. 
International Journal of Computer Science and Engineering Survey (IJCSES), Vol.11, No.3, June 2020

[9] Ekong, V.E., Inyang U.G. \& Onibere E.A, "Intelligent Decision Support System for Depression Diagnosis Based on Neuro-fuzzy-CBR Hybrid," Modern Applied Sciences, pp. vol. 6, issue 7. (ISSN 1913-1844 E-ISSN 1913 - 1852), 2012.

[10] Farrugia, S., Yee, H. \& Nickolls, P, "Implantable Cardiovascular Defibrillator electrogram recognition with a multilayer perceptron," PACE pacing Clinical Electrophysiol, pp. vol. 161, issue 1, pp 28 - 34., 1993.

[11] A. Al-Hajji, "Rule-based Expert System for Diagnosis and Symptoms of Neurological Disorders," Proceedings of ICCIT, 2012.

[12] Singla, J., Grover, D. \& Bhandari, A., "Medical Expert Systems for Diagnosis of various Diseases," International Journal of Computer Application (0975 - 8887), pp. Vol. 93 - No.7, pp36 - 43. ( IJCA: www.ijcaonline.org), May 2014.

[13] Singla, J., Grover D. \& Bhandari A, "Medical Expert Systems for Diagnosis of various Diseases," International Journal of Computer Application(0975 - 8387), pp. vol. 93, issue 7. DOI: 10.5120/16230-5717, 2014.

[14] Kaur, A. \& Kaur A., "Comparison of fuzzy logic and neuro-fuzzy algorithms for air conditioning system," International Journal of Soft Computing and Engineering, pp. Vol. 2, issue 1, March 2012.

[15] A. S. Geetha, "Artificial Neural Networks in Medical Diagnosis," International Journal of Computer Science Issues (IJCSI), pp. Vol. 8, Issue 2, pp 150 - 154. Available online at www.ijcsi.org, 2011.

[16] Obi, J.C. \& Imianvan, A.A., "Interactive neuro-fuzzy expert system for diagnosis of Leukemia," Global Journal of Computer Science and Technology, pp. Vol. 11, issue 12, version 1.0, July 2011.

[17] Omisore, M.O., Samuel, O.W. \& Atajeromavwo, E.J, "A Genetic-Neuro-Fuzzy inferential model for diagnosis of tuberculosis," Applied computing and Informatics, pp. vol.13, pp 27 - 37. http://dx.doi.org/10.1016/j.aci.2015.06.001, 2017.

[18] Nord, J.H. \& Nord, G. D, "MIS Research: Journal Status and Analysis," Information and Management, pp. vol. 29, issue 1, pp 29 - 42., 1995.

[19] Singh, J.; Singh, G; and Singh, A., A Textbook of Artificial Intelligence, New Dehli: UDH Publisher and Distributors (P) Ltd., 2010.

[20] Amato, F., Lopez, A., Pena-Mendez E.M., Vanhara P, Hampl A. \& Havel J, "Artificial neural networks in medical diagnosis.," Journal of Applied Biomedicine., pp. Vol. 11, Issue 3, pp 47 - 58. DOI: 10.2478/v10136-012-0031-x, 2013.

[21] Szolovits, P., Patil, R.S. \& Schwartz W, "Artificial Intelligence in Medical Diagnosis.," Ann. International Medicine, pp. Vol. 108, pp 80 - 87., 1988.

[22] Papik K., Molnar B., Schaefer R., Dombovari Z., Tulassay Z. \& Feher J, "Application of Neural Networks in Medicine - a Review," Medical Science Monitor, pp. vol. 4, issue 3, pp538 - 546., 1998.

[23] Perveen, R., Nabi, M., Memon F.A., Zaman S. \& Ali M, "A Review and Survey of Artificial Neural Network in Medical Sciences," Journal of Advanced Research in Computing and Applications, pp. vol. 3, issue 1, pp 8 - 17., 2016.

[24] W. H. O. (WHO), "Mental Health: Depression," Available at http://www.who.int/mentalhealth/management/depression/definition/en(Accessed(18-12-2-18), 2010.

[25] Leon ,B.S., Alanis A.Y., Sanchez E., Ornelas-Tellez, F. \& Ruiz-Velazquez, E. , "Inverse Optimal Neural Control of blood glucose level for type I diabetes Mellitus Patients," Journal of Franklin, pp. vol. 349, pp 1851 - 1870., 2012.

[26] Alade O.M., Sowunmi O.Y. Misra S., Maskelionos R. \& Damasevicius R, "A Neural Network Expert System for the Diagnosis," 2018.

[27] Sadi, S., Maleki, A., Hashemi, R., Panbechi, Z. \& Chalabi, K, "Comparison Algorithms in the Diagnosis of Type II Diabetes," International Journal of Computational Science \& Applications (IJCSA), pp. vol. 5, issue 5, pp 1 - 12.

[28] Myers, J., Souza, C.R.D., Borghi-Silva, A., Guazzi, M., Chase, P., Bensimhon, D., Peberdy, M.A., Ashley, E., West, E., Cahalin, L.P., Forman, D. \& Arena, R, "A Neural Network Approach to predicting outcomes in heart failure using cardiopulmonary exercise testing," International Journal of Cardiology, pp. vol. 171, pp 265 - 269. http://dx.doi.org/10.1016/j.ijcard.2013.12.031, 2014.

[29] Atkov, O.Y., Gorokhova, S.G., Sboev A.G., Generozov E.V., Moroshkina, S.Y. \& Cherney, N.N, Journal of Cardiology, pp. vol. 59, pp 190 - 194. (Available online at www.sciencedirect.com), 2012.

[30] Miao K.H. \& Miao J.H, "Coronary Heart Disease Diagnosis using Deep Neural Networks," International Journal of Advanced Computer Science and Applications., pp. vol. 9, No 10, pp 1 - 8 ., 2018. 
[31] Acharya, U.R., Oh, S.L., Hagiwara, Y., Tan, T.H., Adam M. Gertych A. \& San T.S, "A deep Convolutional Neural Network model to classify heartbeats.," Computes in Biology and Medicine, p. DOI:10.1016/j.compbiomed.2017.08.022, 2017.

[32] Acharya U.R., Fujita H., Hagiwara Y., Tan, J.H. \& Adam M, "Application of Deep Convolutional Neural Network for automated detection of myocardial infarction using ECG signals," Information Sciences, pp. p 190 - 198. http://dx.doi.org/10.1016/j.ins.2017.06.027 (Available at ScienceDirect), 2017.

[33] Ting, F.F., Tan, Y.J. \& Sim, K.S, "Convolutional neural network improvement for Breast Cancer Classification," Exert SYstems with Applications, p. doi:http://doi.org/10.1016/j.eswa.2018.11.008, 2018.

[34] Huo, Q.; Bing, Z.; Hu, C.; Li, M.; Yang, K.; Mo, Z.; Xie, X.; Liao, J.; Lu, Y.; Horie, S.; and Lou, M., " RankProb Combined with Genetic Algorithm Optimized Artificial Neural Network Establishes a Diagnostic and Prognostic Prediction Model that Revealed".

[35] Naushad, S.M.; Ramaiah, M.J.; Pavithrakumari, M.; Jayapriva, J.; Hussain, T.; Alrokayan, S>A.; ...; Kutala, V.K., "Artificial Neural Network - based exploration of gene-nutrient interaction in folate and Xenophobic Metabolic Pathways that modulate Susceptibility to breast cancer," pp. vol. 580, issue 2, pp 159 - 168. DOI: 10.1016/j.gene.2016.01.023, 2016.

[36] Shell, J.; and Gregory, W.D., "Efficient Cancer Detection using Multiple neural networks," IEEE Journal of Transactional Engineering in Health and Medicine, pp. vol. 5, pp 1 - 37. DOI: 10.1109/JTEHM.2017.2757471, 2017.

[37] Pergialiotis, V., Pouliakis, A., Parthenis, C., Damaskou, V., Chrelias, C., Papantoniou, N. \& Panayiotides, I, "The Utility of Artificial neural networks and classification and regression trees for the prediction of endometrial cancer in postmenopausal women," Public Health, pp. vol. 164, pp 1 6., 2018.

[38] Ziynet , Y.M. \& Bozkurt, R, "Determination of women iron Deficiency ANemia using Neural networks Networks.," Journal of medical systems, pp. vol. 36, pp 2941 - 2945. DOI:10.1007/s10916011-9772-4, 2012.

[39] Neha, S.; Vikas, K.; and Ashish, L., "An Intelligent system based on backpropagation neural network and particle swarm optimization based on neural network for diagnosing Anemia in pregnant ladies," i-manager's Journal on information technology, pp. vol. 6, issue 2, pp 27 - 32 . https://doi.org/10.26634/jit.6.2.13574, n.d.

[40] Parveen, R., Jalbani, A.H., Shaikh, M. Memon, K.H., Siraj, S. Nabi, M. \& Lakho, S, "Prediction of Malaria using Artificial neural networks.," International Journal of Computer science and Network Security, pp. vol. 17, No 12, pp 79 -89., 2017.

[41] Pandit P. \& Anand, A, "Artificial neural networks for detection of Malaria in RBCs".

[42] Lyu, J. \& Zhang, J, "BP Neural Network prediction model for suicide attempts among Chinese rural residents," Journal of Affect Disorder, pp. vol. 246, pp 245 - 473. https://doi.org/10.1016/j.jad.2018.12.111, 2019.

[43] Huang, K.; Wu, C. and Su, M., "Attention-based convolutional neural network and long-short memory for short-term detection of mood disorder based on elicited speech responses," Pattern Recognition, pp. vol. 88, pp 668 - 678, 2019.

[44] Fonseca M.B., Andrades, R.S.D., Bach, S.D.L., Wiener, C.D. \& Oses, J.P, "Bipolar and Schizophrenia Disorders Diagnosis using Artificial Neural Network," Neuroscience \& Medicine, pp. vol. 9, pp 209 - 220. DOI: 10.4236/nm.2018.94021, 2018.

[45] Basaia, S.; Agosta, F.; Wagner, L.; Canu, E.; Magnani, G.; Santangelo, R.; and Filippi, M., "Automated classification of Alzheimer's disease and mild cognitive impairment using a single MRI and deep neural networks," Journal of NeuroImage Clinical, p. vol. 21. https: doi.org/10.1016/j.nicl.2018, 2019.

[46] Bansal, D., Chhikara, R., Khanna, K., \& Gupta, P, "Comparative Algorithm for Detecting various Machine Learning Algorithm for Detecting Dementia," Procedia Computer Science, pp. vol. 132, pp 1497 - 1502. DOI:10.1016/j.procs.2018.05.102, 2018.

[47] Zeng, L., Wang, H., Hu, P., Yang, B., Pu, W., Shen, H., Chen, X., Liu, Z., Yin, H., Tan, Q., Wang, K.S., \& Hu, D, "Multi-site Diagnostic Classification of Schizophrenia using Discriminant Deep Learning with Functional Connectivity MRI," EBioMedicine, pp. vol., pp $74 \quad-85$. https://doi.org/10.1016/j.ebiom.2018.03.017, 2018. 
International Journal of Computer Science and Engineering Survey (IJCSES), Vol.11, No.3, June 2020

[48] Chattopadhyay S., Kaur ., Rabhi F., \& Acharya, U.R, "Neural Network Approaches to Grade Adult Depression," Journal of Medical Systems, pp. vol. 36, pp2803 - 2815. DOI: 10.1007//s10916-0119759-1, 2012.

[49] Ojeme B. \& Mbogho A, "Selecting Learning Algorithms for Simultaneous Identification of Depression and Comorbid Disorders.," Procedia Computer Science, pp. vol. 96, pp 294 - 303. DOI: 10.1016/j.procs.2016.08.174 (Available online at www.sciencedirect.com), 2016.

[50] Ojeme B., Akazue M. \& Nwelih E, "Automatic Diagnosis of Depressive Disorders using Ensemble Techniques.," African Journal of Computing \& ICT, pp. vol. 8, issue 3(2), pp 31 - 37., 2016.

[51] Guntuku, S.C., Yaden, D.B., Kern, M.L., Ungar, L.H. \& Eichstaedt, J.C, Detecting depression and mental illness on social media: an integrated review, 2017: vol. 18, pp 43 - 49. http:dx.doi.org/10.1016/j.cobeha.2017.07.005, Current Opinion in Behavioural Sciences. 